\title{
Immunohistochemical response in rats of beta-tricalcium phosphate (TCP) with or without BMP-2 in the production of collagen matrix critical defects
}

\author{
Eloá Rodrigues Luvizuto a , Júlio César Silva de Oliveira ${ }^{a}$, \\ Pedro Henrique Silva Gomes-Ferreira ${ }^{a, *}$, Cassiano Costa Silva Pereira ${ }^{a}$, \\ Leonardo Perez Faverani $^{\mathrm{a}}$, Cristina Antoniali ${ }^{\mathrm{b}}$, Roberta Okamoto ${ }^{\mathrm{b}, 1}$ \\ a Department of Surgery and Integrated Clinic, Sao Paulo State University (UNESP), School of Dentistry, Araçatuba, Brazil \\ ${ }^{\mathrm{b}}$ Department of Basic Sciences, Sao Paulo State University (UNESP), School of Dentistry, Araçatuba, Brazil
}

\section{A R T I C L E I N F O}

\section{Article history:}

Received 12 July 2016

Received in revised form 2 February 2017

Accepted 24 February 2017

\section{Keywords:}

BMP

Collagen matrix

Histologic evaluation

Immunohistochemistry

\begin{abstract}
A B S T R A C T
This study aimed to assess the biological response of BMP-2 (bone morphogenetic protein-2) in supplementation with $\beta$-tricalcium phosphate (TCP) as a carrier in the bone healing of surgical defects in rats' calvaria. A critical-size defect ( $5 \mathrm{~mm}$ in diameter) was filled with $\beta$-TCP alone or added with that plus $5 \mathrm{mg}$ of BMP-2 at 5,15 , and 30 postoperative days. Histomorphometric and immunohistochemical (osteocalcin, collagen type I, and metalloproteinase-9) analysis was performed to assess the features of bone healing. Histological behavior and collagen type I labeling showed increased formation of the collagen matrix, leading to a higher percentage of newly formed bone and biomaterial for tissue and more total mineralization of pure TCP when compared to the other groups. The supplementation with BMP-2 promoted faster TCP remodeling; however, there was no statistically significant difference for the bone formed in both groups $(P>0.05)$. Collagen-matrix formation and new bone formation reached maximum levels when the defects were filled with pure TCP, even exceeding the levels from BMP-2 supplementation.
\end{abstract}

(c) 2017 Elsevier GmbH. All rights reserved.

\section{Introduction}

The absence of the alveolar bone is one of the major challenges in rehabilitation after dental implants are installed, and in some cases, subsequent surgical procedures are necessary. Autogenous bone remains the gold standard (Boyne and James, 1980; Tatum, 1986; Misch, 1987) for the reconstruction of defects caused by disease and trauma (Tessier et al., 2005), as it enables the placement of implants (Brånemark et al., 1975; Wada et al., 2001; Proussaefs and Lozada, 2005; Esposito et al., 2006). Despite possessing all the desirable properties, autogenous bone has some disadvantages, such as donor site morbidity, limited quantity, and a need for general anesthesia in extraoral grafts.

With the extensive development of tissue engineering, bone substitutes are achieving bone scaffolds with properties similar to

\footnotetext{
* Corresponding author at: Department of Surgery and Integrated Clinic, Sao Paulo State University (UNESP), School of Dentistry, Araçatuba, Brazil.

E-mail address: pedroferreirabmf@gmail.com (P.H.S. Gomes-Ferreira).

1 FAPESP (\#2008/03291-8).
}

those of autogenous bone, especially osteoconductive properties, which favor the process of bone formation (Yao et al., 2005). In this scenario, there is a matrix of $\beta$-tricalcium phosphate (TCP), which is carrier bone and is the target of several studies.

Along with the discovered bone scaffolds, the potential osteoinductive bone morphogenetic proteins (BMPs) are produced by osteoblasts, which stimulate the differentiation of undifferentiated mesenchymal cells into chondrocytes (Wozney and Rosen, 1998; Reddi, 1998). Several studies (Wozney and Rosen, 1998) have confirmed the importance of bone morphogenetic protein-2 (BMP-2) in cell differentiation and bone repair.

The study of the cellular and molecular mechanisms involved in bone repair can be better understood and justified by immunohistochemical techniques. In the process of bone formation, osteocalcin signals bone mineralization and collagen type I (Col I). These are products of osteoblasts (the main components of the organic matrix of bone tissue), and they are produced and released during bone formation. The use of extracellular matrix components, such as Col I- and chondroitin sulfate-enhanced osteogenic 
differentiation and bone-matrix accumulation, was found in some studies (Rentsch, 2014).

On the other hand, the answer depends on bone metabolism, such as the bone resorption by osteoclasts that produce controlled proteases. These proteases include the metalloproteinase-9 (MMP9) enzyme, indicating the degradation of the extracellular matrix, which is replaced by new bone. Matrix metalloproteinases are a family of proteolytic enzymes produced by inflammatory cells, fibroblasts, and endothelial cells. The enzymes cleave to extracellular matrix components and a wide range of bioactive molecules that are involved in wound healing (Carlson et al., 2013).

Considering the dynamics of bone healing and the importance of scientific knowledge of biomaterial, it is necessary and appropriate to carry out histologic and immunohistochemical studies. These studies will further elucidate the performance of bone substitutes, including TCP, which are associated with the use of carriers such as BMP-2 in regenerative techniques and with the expression of bone matrix proteins as markers in the bone process.

Therefore, the present study looks at the biological role of TCP, whether supplemented with BMP-2 or not, in the synthesis of collagen matrices in surgically created cavities within rat calvaria.

\section{Material and methods}

\subsection{Study design and ethics}

This present study is in accordance with the principles of laboratory animal care and national laws on animal use, and it was authorized by the Animal Research Ethics Committee of the São Paulo State University, Brazil (protocol \#2008-004517). We purchased the 45 Wistar male adult rats (90 days old) used for this study from the Animal Center of São Paulo State University and maintained them at a temperature of $22^{\circ} \mathrm{C}$ under a $12 \mathrm{~h}$ light $/ 12 \mathrm{~h}$ dark cycle, with free access to water and rodent food. A total of 45 calvarial defects ( $5 \mathrm{~mm}$ in diameter) were randomly divided into 3 groups, with a total of 5 defects per treatment group $(n=5)$, and into 3 evaluation times $(5,15$, and 30 days). The treatment groups were established as follows: (1) 500-1000 mm $\beta$-TCP (Cerasorb M Curassan Ltd., Germany), (2) TCP plus 5 mg BMP-2 (R \& D Systems, Inc., Minneapolis, MN, USA), and (3) the empty control (untreated group). Our group used the same experimental design and evaluation groups in our previous studies (Luvizuto et al., 2011, 2012).

\subsection{Surgical procedures}

After general anesthesia with xylazine $(0.03 \mathrm{~mL} / 100 \mathrm{~g}$ body weight [bw]/intraperitoneal [ip]; Dopaser Laboratories Calier SA, Barcelona, Spain) and ketamine ( $7 \mu \mathrm{L} / \mathrm{kg}$ bw/ip; Fort Dodge Saúde Animal Ltd., Brazil), trichotomies of the animals' skulls were completed, disinfecting with polyvinylpyrrolidone iodide (PVPI 10\%, Riodeine Degermante, Rioquímica, São José do Rio Preto, SP, Brazil). Using aseptic techniques, an incision was made through the skin and periosteum of the skull, and a full-thickness flap was obtained. A defect of $5 \mathrm{~mm}$ diameter was prepared in each animal's parietal region with a bone trephine drill (3i Implant Innovations Inc., Palm Beach Gardens, USA) under copious saline irrigation.

The defect was treated as described above prior to the repositioning of the periosteum and sutured with polyglactin 910 (Vycril 5.0, Ethicon, Johnson Prod., São José dos Campos, Brazil). The skin was sutured with nylon (Nylon 5.0, Mononylon, Ethicon). All animals received a single dose of 20,000 IU of benzathine penicillin (Pentabiotic, Veterinário Pequeno Forte, Fort Dodge Animal Health Ltd., Campinas, Brazil) intramuscularly. The rats were euthanized with anesthetic overdose (Sodic Thiopental, $150 \mathrm{mg} / \mathrm{kg}$ ) after 5, 15, or 30 postoperative days.

\subsection{Sample processing}

The skull parts in the study were fixed in formalin solution, underwent decalcification in EDTA (18\%), and were then dehydrated using a series of alcohols. After these steps, they were cleared with xylene, embedded in paraffin, and cut to obtain thicknesses of $5 \mu \mathrm{m}$ for mounting on slides. The pair slides were stained with hematoxylin and eosin (HE) (Merck \& Co., Inc.), and the odd slides were then prepared for immunohistochemistry analysis.

\subsection{Histomorphometric analysis}

The measurements were performed using an optical microscope coupled with a camera image capture (JVC TK 1270 Color Video Camera) lens (with $4 \times$ increase); the microscope was connected to a computer that had a software analyzer to scan images (Leica Qwin Color/RGB). The scanned images were saved as JPEG files, analyzed, and projected on a monitor (SyncMaster 3NE, 15 inches). All slides were encoded, and the observer performing the evaluation did not know which group the blades belonged to. There were 3 fields in each histological section. These fields corresponded to the areas of bone tissue present in the central region (1 field) and the peripheral regions ( 2 fields) of the bone defects. The fields were standardized through a toll straight from the RGB software. First, the images from the defect were measured in all dimensions (from one side to another side). Thus, the images were divided into 3 parts to show the 2 peripheral regions and the central region.

The data obtained in this study were transformed into absolute values of pixels to get the relative percentage values so as to minimize the interference of the size difference. The data obtained were (1) the total volume of new bone, (2) the total volume of biomaterial, and (3) the total volume of mineralized tissue. Each was expressed as a percentage of the total volume of tissue.

\subsection{Statistical analysis}

The data were analyzed through multiple comparisons of the means using a 2-way ANOVA and a Tukey posttest. The difference was attributed to a $P<0.05$.

\subsection{Immunohistochemical analysis}

Immunoperoxidase was used for detection. The endogenous peroxidase activity was inhibited with hydrogen peroxide. Following this, the blades passed though the stage of antigen retrieval, with a citrate phosphate buffer ( $\mathrm{pH}$ 6.0). The primary polyclonal antibodies produced in goats were those that act against osteocalcin (OCN; SC-365797, Santa Cruz Biotechnology, Dallas, TX, USA), Col I (SC-8785), and MMP-9 (SC-6840), with the aim of analyzing the formation and degradation of the organic matrix. We used a polyclonal biotinylated secondary goat antibody that is produced in rabbits (Pierce Biotechnology, Waltham, MA, USA) and an Avidin and Biotin amplifier kit (Vector Laboratories, Burlingame, CA, USA). The chromogen used was diaminobenzidine (Dako, Santa Clara, CA, USA), and the end of the reaction was carried out against the cutstaining with the Harris hematoxylin. For each of the antibodies used, we evaluated the expression of these proteins semiquantitatively by assigning scores. The analysis was performed under a light microscope (Leica DMLB, Heerbrugg, Switzerland). Negative controls were performed to assess the specificity of the antibodies. Absence of immunostaining was observed when the primary antibody was substituted with the serum of the host species, acting as a negative control for the secondary antibody. All lab processing was standardized based on previous studies (Luvizuto et al., 2011, 2012). 


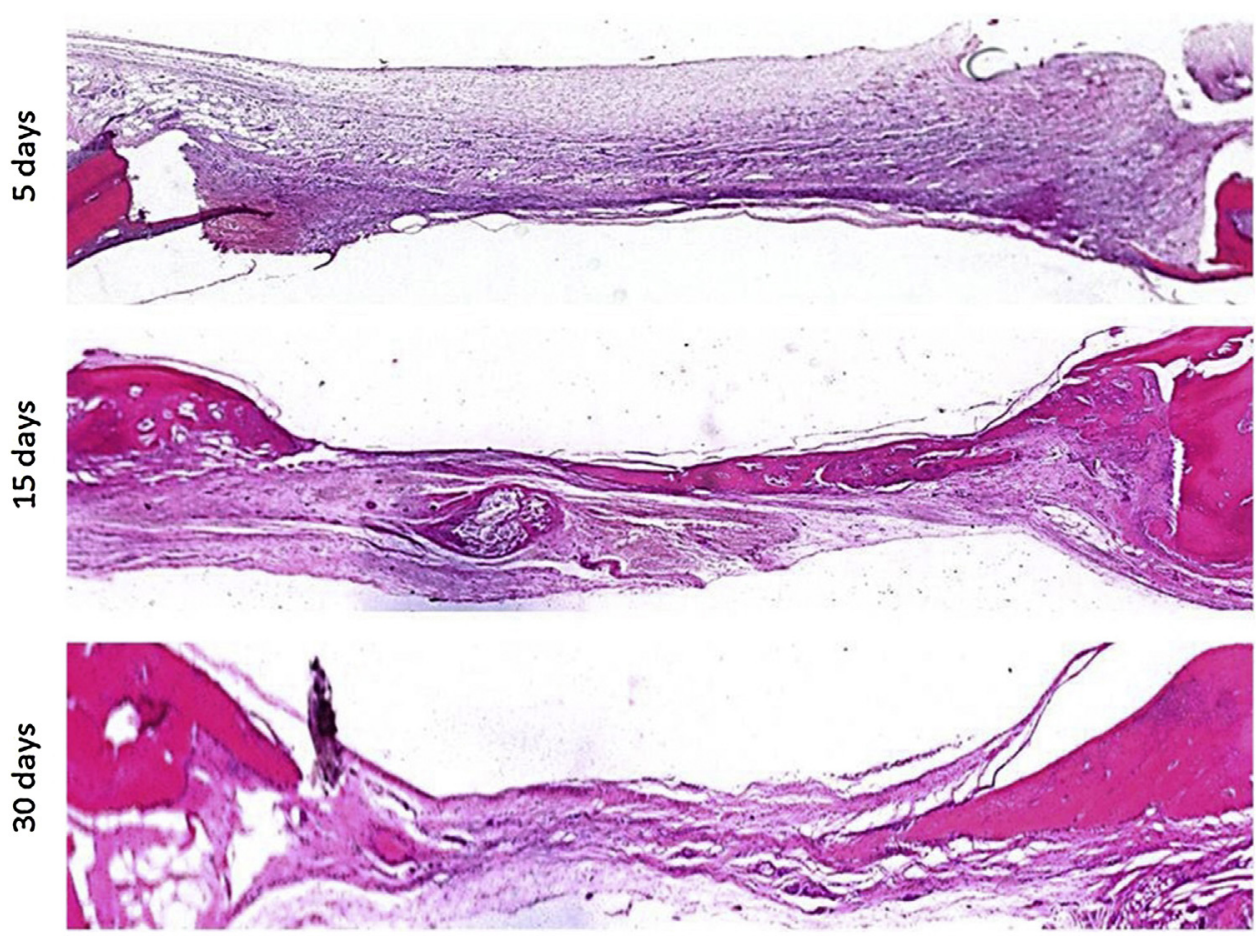

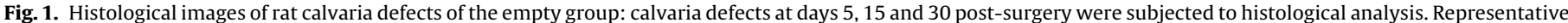

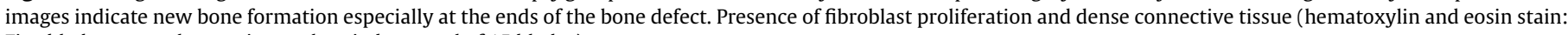
Five blades per each experimental period - a total of 15 blades).

\section{Results}

\subsection{Histology}

\subsubsection{Empty control (untreated group) (Fig. 1)}

At the initial time point (day 5), the presence of newly formed tissue with a discrete number of fibroblasts was observed throughout the surgical site, as were some lymphocytes and macrophages. In some specimens, there was slight new bone formation along the edges of the surgical cavity.

At day 15, in some specimens, a thin strip of new bone was revealed, occupying a large extent of the surgical cavity. In other cases, the bone filled a lesser extent above the surgical site. In terms of differentiation, the bone tissue exhibited no discrete number of fibroblasts, macrophages, or lymphocytes.

At day 30, in most specimens, a thin strip of new bone partially occupying the surgical site was observed. In some cases, there was slight bone formation near the edge of the surgical cavity. The rest of the space was found to be occupied by connective tissue with a discrete number of fibroblasts. In one of the specimens, a thicker bone trabeculae partially filled the surgical cavity.

\subsubsection{TCP group (Fig. 2)}

At the initial time point (day 5), there was infiltration, with inflammatory cells, fibroblasts, and connective tissue around the material.

At day 15, bone formation was observed mainly at the ends of the bone defect, but also from the biomaterial granules. In some tissue regions, wrapping of the biomaterial was observed.

At day 30, maturation of newly formed bone tissue between the granules of the biomaterial and the bone defect edges was observed.

\subsubsection{TCP plus BMP-2 group (Fig. 3 )}

At all times (days 5, 15, and 30), similar results were observed when compared with the isolated use of TCP. Thus, higher bone formation was not shown with the addition of BMP-2 (compared to with TCP alone).

\subsection{Histometry}

All groups showed increased bone formation during the analyzed periods. The TCP group showed a greater amount of new bone relative to total tissue in all periods (with statistical significance) when compared to the TCP + BMP-2 and empty control groups at 5 and 15 days (Graph 1 ). Regarding the amount of biomaterial relative to total tissue, the TCP group showed higher levels when compared to the TCP + BMP-2 group, but this was not statistically significant (Graph 2).

In relation to amount of mineralized tissue relative to total tissue, the TCP group showed the greatest amount of the 3 groups (Graph 3 ). The TCP and TCP + BMP-2 groups had superior results to those of the empty group, and this difference was statistically significant for all periods (days 5, 15, and 30). However, the TCP group achieved statistically significant results compared to the TCP + BMP-2 group at 15 days.

\subsection{Immunohistochemistry}

Semiquantitative analyses of immunoblots was performed by assigning scores, where 0 represented the absence of marking, 1 represented mild or moderate marking, and 2 or 3 represented intense labeling. The osteoblastic cell lines were immunostained for all cuts to show cell viability in the assessed bone defects.

Osteocalcin is a protein that characterizes the osteoblast phenotype; the mineralization process at 5 days showed mild labeling of this protein in the TCP group. At 15 and 30 days, the osteocal- 

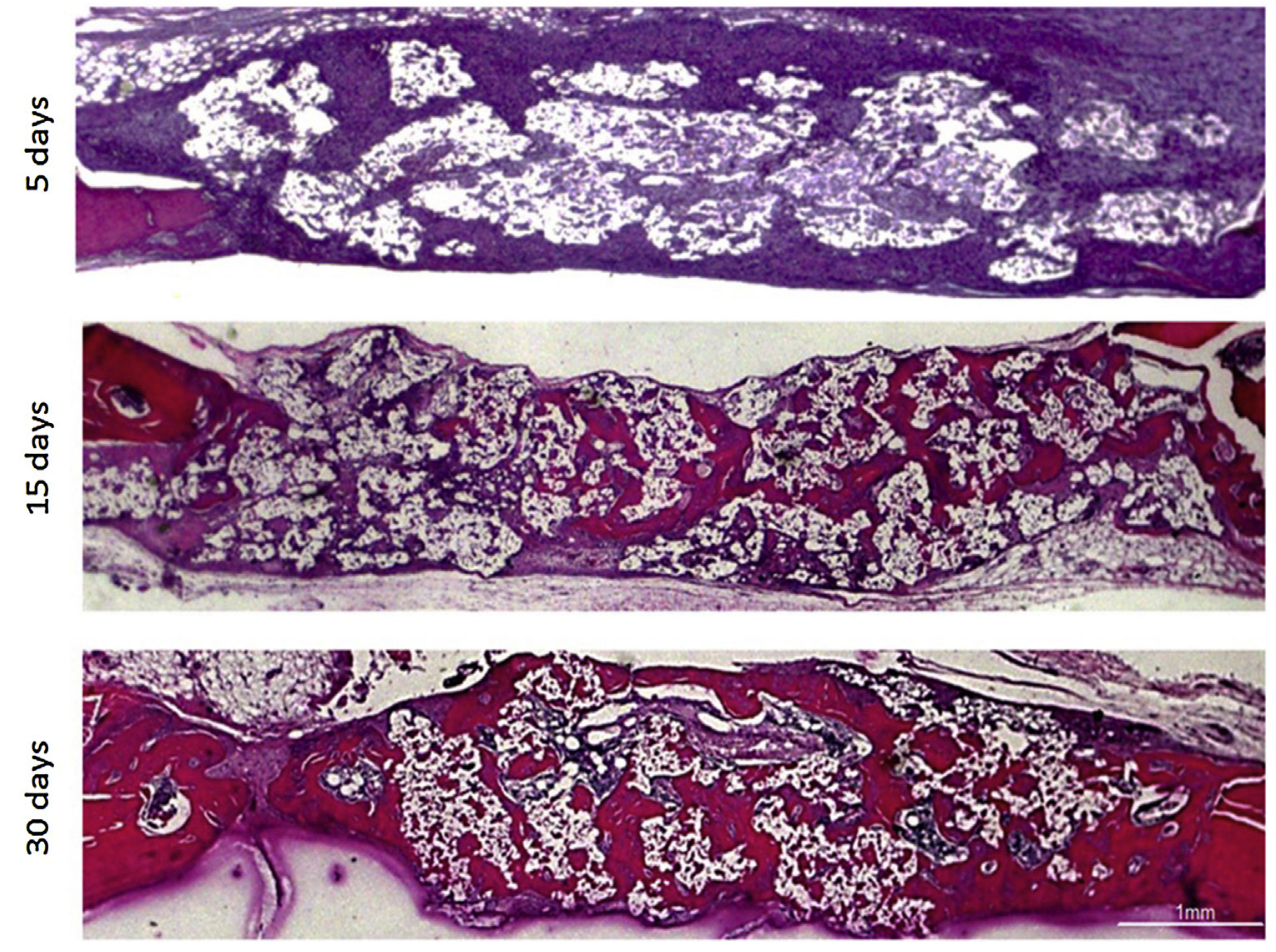

Fig. 2. Histological images of rat calvaria defects treated with $\beta$-TCP alone: calvaria defects at days 5,15 and 30 post-surgery were subjected to histological analysis. Representative images indicate that complete defect closure was achieved (hematoxylin and eosin stain: Five blades per each experimental period - a total of 15 blades).
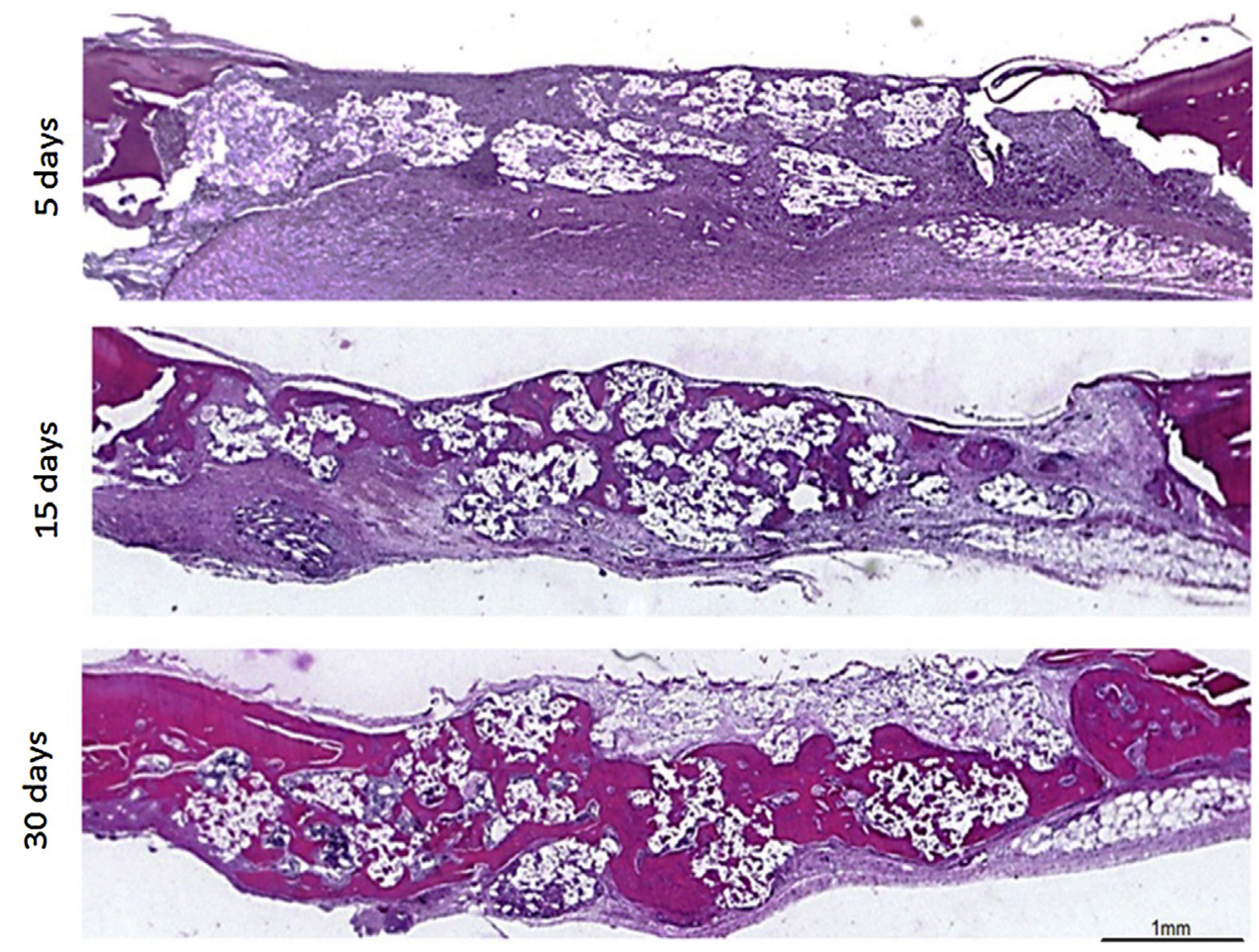

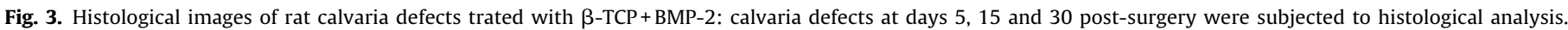
Representative images indicate that complete defect closure was achieved (hematoxylin and eosin stain: Five blades per each experimental period - a total of 15 blades).

cin was evident but had moderate labeling. For the BMP-2 group at 15 days, osteocalcin appeared to have less marking, but it had moderate labeling at 30 days (Fig. 4).

Col I, the main protein of the organic matrix, was introduced and expressed in all the cuts, with positive staining for fibroblasts, osteoblasts, the extracellular matrix, and the mineralized bone tissue. The predominance of the TCP immunostaining group in both the 15- and 30-day periods corroborates the results observed in the histomorphometric analysis. With the addition of BMP-2, the expression of Col I was more evident at 15 days and in the untreated 

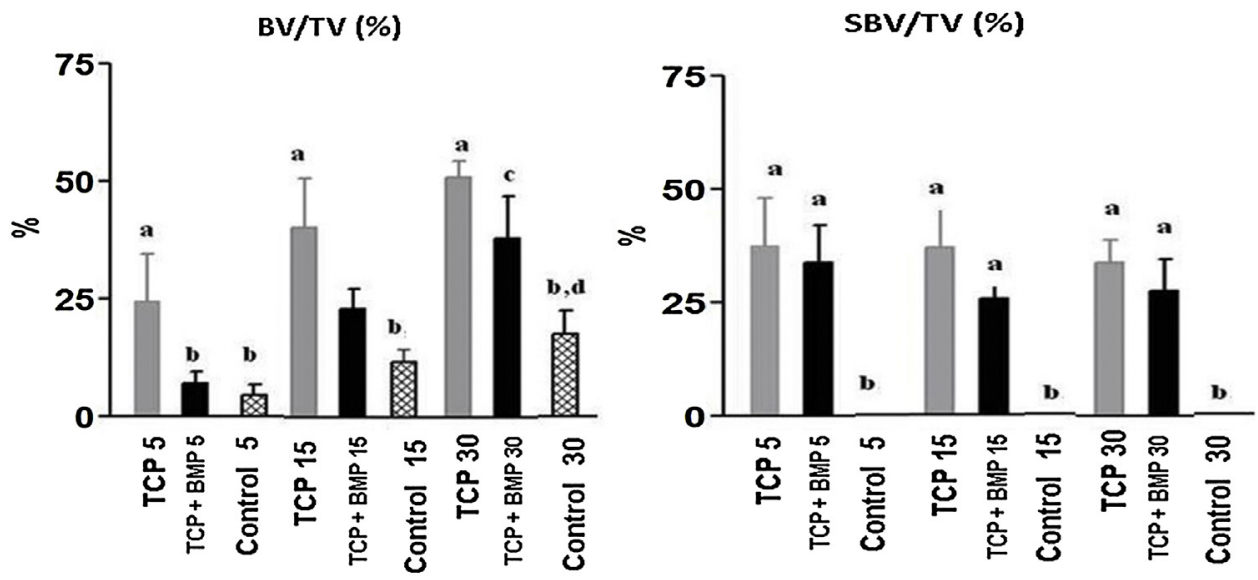

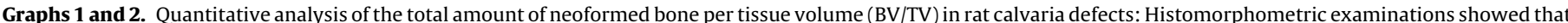

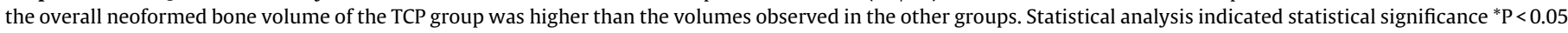

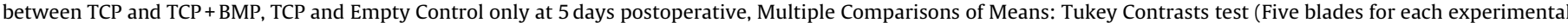

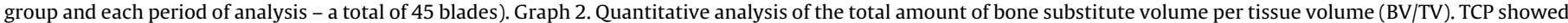

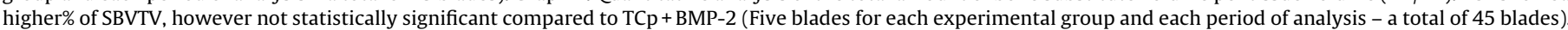
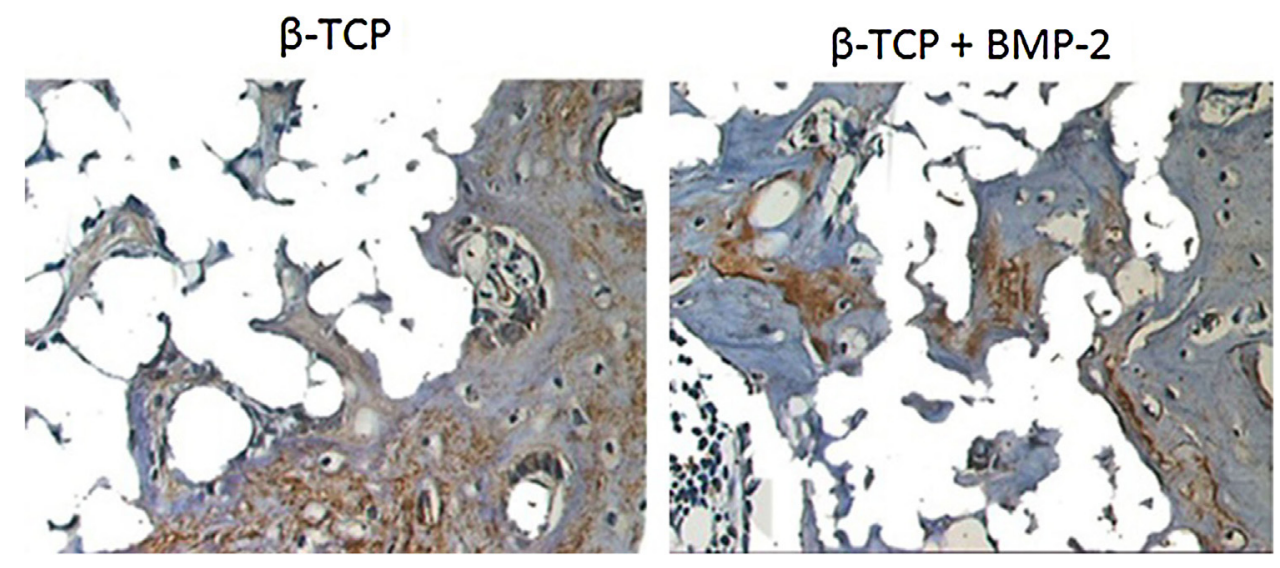

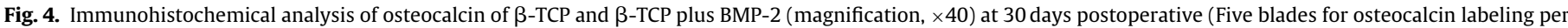
each experimental group: $\beta$-TCP and $\beta$-TCP plus BMP-2 - a total of 10 blades).

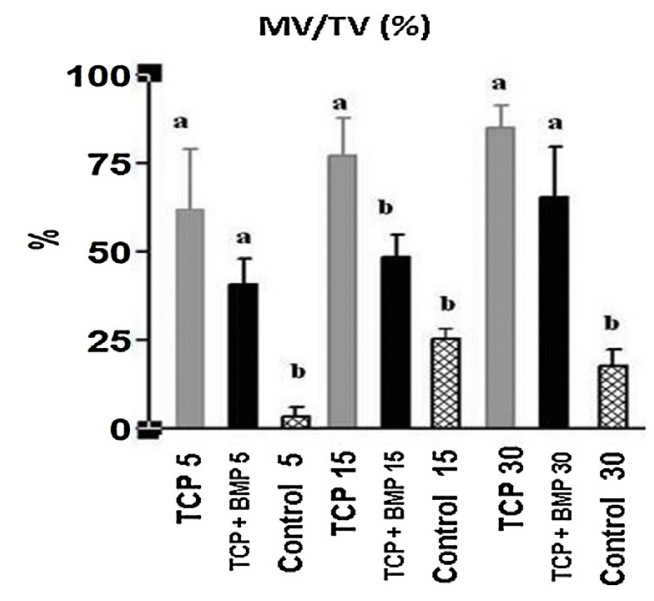

Graph 3. Quantitative analysis of the total amount of mineral tissue volume per tissue volume (MV/TV) in rat calvaria defects: Histomorphometric examinations showed that the overall mineralized volume of the TCP group was higher than the volumes observed in the other groups. Statistical analysis indicated statistical significance ${ }^{*} \mathrm{P}<0.05$ between TCP and TCP + BMP, TCP and Empty Control at 15 postoperative days. Multiple Comparisons of Means: Tukey Contrasts test (Five blades for each experimental group and each period of analysis - a total of 45 blades). group. This protein had lower levels than were observed in the other study groups (Graph 4 ).

MMP-9 signals the degradation of the extracellular matrix and its replacement by newly formed bone tissue; it was also observed in all periods, with positive staining for osteoclasts. Intense immunostaining was observed in the TCP + BMP-2 group for all periods $(5,15$, and 30 days), especially at 30 days (Graph 5). In the TCP and empty-marking groups, the observed effects were mild (at 15 and 30 days) or moderate (at 5 days).

\section{Discussion}

Bone repair capacity is limited, and in many cases, defects in reconstruction occur following trauma, oncological resections, extractions, infection, or the prior installation of dental implants (Brånemark et al., 1975; Esposito et al., 2006). Since they have some disadvantages, autografts have been replaced by biomaterials of either natural or synthetic origin; these materials are being widely studied and disseminated.

The bioactive ceramic $\beta$-TCP has excellent biological and structural properties. It is a resorbable material and is capable of supporting bone formation, as demonstrated in both animal models (Bostrom et al., 1995; Wozney and Rosen, 1998; Luvizuto et al., 2011, 2012) and clinical trials (Wozney and Rosen, 1998; Reddi, 


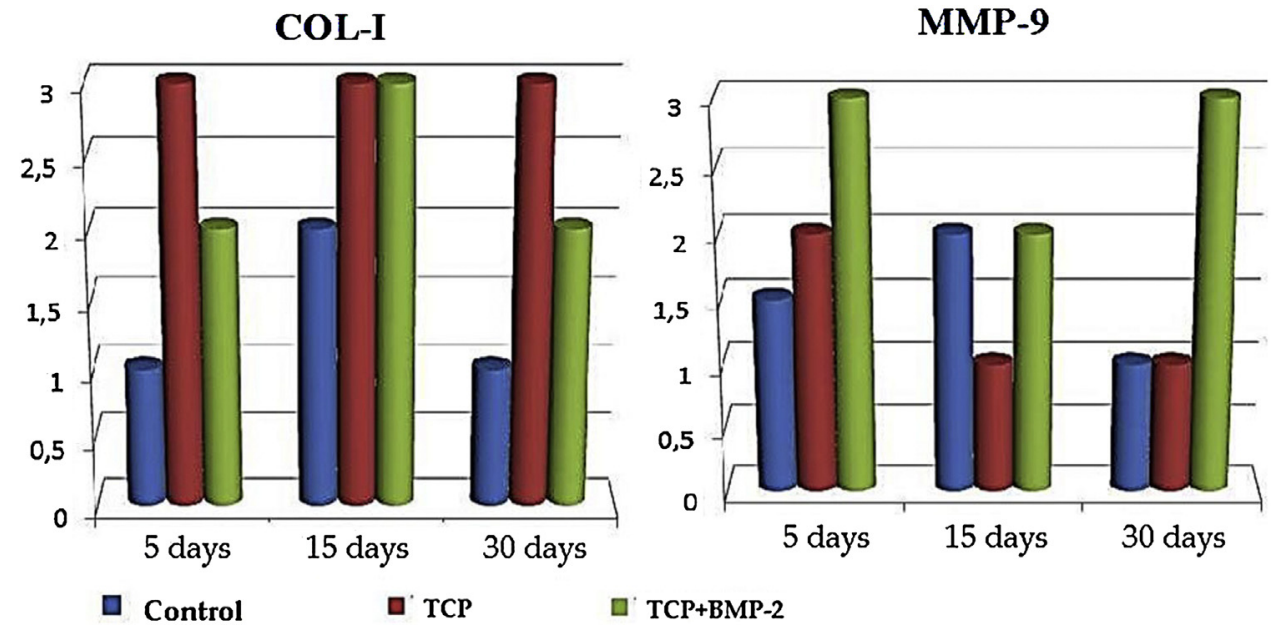

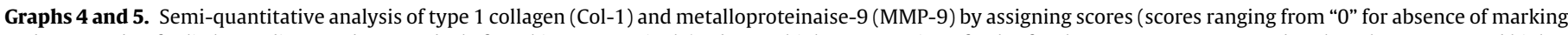

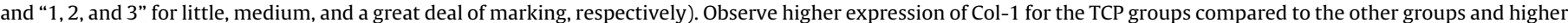

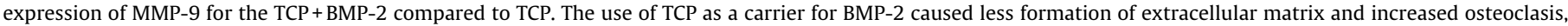

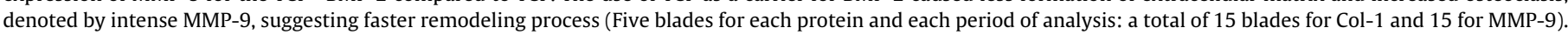

1998). Histological examination showed that, at periods of 5, 15, and 30 days, its excellent osteoconductive ability translated to a higher percentage of newly formed bone and mineralized tissue relative to total tissue. The examination also showed a higher percentage of this biomaterial in regenerated tissue, which can suggest delayed resorption relative to that of TCP + BMP-2.

An important aspect of tissue repair and the maximization of newly formed bone mineralization is the use of growth factors. BMP-2 acts as a local regulator of cellular activity and is a potent mitogenic agent. However, to obtain the effectiveness of local application, BMP-2 requires an efficient carrier that is biocompatible, osteoconductive, and resorbable (Bostrom et al., 1995). In this sense, new therapies and concepts have been implemented to maximize the process of bone formation after reconstruction. Among these is the use of osteoconductive biomaterials that serve as carriers of BMPs. However, doubts have arisen about which biomaterial is suitable for this purpose and for supplementation with BMP-2, which provides a substantial advantage to the overall process of consolidating the biomaterial.

The present study demonstrated the excellent osteoconductive properties of TCP, regardless of the presence of BMP-2, confirming previous results from our laboratory (Luvizuto et al., 2011). Specifically, the results strongly suggest that pure TCP induces complete mineralization of critical-size defects in rat calvaria. This fact is confirmed by the closure of the bone defect at 30 days.

The results are important because they show that the TCP group had similar performance to the TCP + BMP-2 group. TCP's osteoconductive properties were not as enhanced with the addition of BMP-2 as they were in the previous experimental study (Bostrom et al., 1995). Moreover, it is a growth factor, and a high cost is associated with side effects such as swelling and foreign body response. Thus, in the present experimental conditions, our data confirm that TCP is not the ideal carrier for BMP-2 (Luvizuto et al., 2011, 2012).

The supplementation of BMP-2 with TCP, both at the dose used in this study ( $5 \mathrm{mg}$ ) and at higher doses (such as15 mg), showed results similar to those of autografts (Van Den Bergh et al., 2000). This finding is consistent with our study, in which supplementing TCP with BMP-2 did not enhance bone formation. Only TCP loaded with a much larger dose of BMP-2 showed an increase in bone formation that was satisfactory in relation to that of pure TCP (Toriumi and Robertson, 1993; Van Den Bergh et al., 2000; Maus et al., 2008; Dohzono et al., 2009). Moreover, the supplementation of TCP with PTH does not improve bone formation compared to
TCP alone (Sohier et al., 2010). Previous studies have shown better results after supplementation with BMP-2; however, the observed effects are dose-dependent (Johnson et al., 1998; Schliephake et al., 2008; Bai et al., 2009; Zhao et al., 2010; Yun et al., 2010).

According to data from an in vitro study that examined the dosedependency of BMP-2 (Toriumi and Robertson, 1993), a titration of $200 \mathrm{mg}$ was effective and active. However, in that study, it was not possible to separately evaluate the release kinetics of BMP-2 and those of the carrier TCP.

As observed in the results of this research, it can be suggested that the lack of differences between BMP-2 used with TCP and TCP used alone is due to the activity of bone morphogenetic protein pathways activated by osteoclasts when there is a triggered or accelerated activity of bone remodeling compartments. This in turn results in greater osteoblastic activity. Data from the literature support this statement, whereby the addition of BMP-2 to TCP leads to more rapid bone remodeling of defects that is found with pure TCP (Van Den Bergh et al., 2000).

The effect of BMP-2 in osteoclasts was investigated (Seeherman et al., 2006) to demonstrate that BMP-2 induced the differentiation of progenitor cells into mature osteoclasts in cultured rabbit cells, thus increasing the expression of messenger RNA for cathepsin $\mathrm{K}$ and carbonic anhydrase II. Both of these are essential enzymes for the degradation of bone's organic compounds and inorganic matrices. Our study could explain the lesser amount of bone formation and mineralization in the TCP + BMP-2 group compared to the pure TCP group. Increased osteoclast-induced BMP-2 probably led to the bone resorption observed in this group. Therefore, it is important to evaluate longer postoperative periods than those used in this study so as to evaluate the long-term quantitative and qualitative changes that occur in the bone formed within the defect.

It is suggested that TCP is a biomaterial that can perform as well as, or even better than, autogenous bone graft particles in bone regeneration. This also suggests that, in a clinical setting, TCP can provide osteoconductive properties similar to those of autogenous bone. Furthermore, the potential clinical importance is that TCP's osteoconductive properties as a carrier were not improved with the indicated dosage of BMP-2, suggesting that further efforts to improve the clinical parameters of bone formation are required.

Regarding the immunostained proteins, osteocalcin and Col I expression were moderate and intense in the TCP group for all periods, especially at 15 and 30 postoperative days; these were translated into the remarkable formation of extracellular matrices, 
which serve as scaffolds for proliferation and deposition of a mineralized matrix of osteoblastic cells. Relative to the empty group, light immunostaining was observed with the addition of BMP-2 (BMP$2+\mathrm{TCP}$ ), and there was moderate to intense immunolabeling for this protein when compared to that of pure TCP. This finding implies that BMP-2 did not enhance the formation of collagen matrices with the TCP framework, thus resulting in a lower percentage of mineralized tissue compared to pure TCP.

The MMPs are the largest family of enzymes that degrade extracellular matrix components and that thus control bone regeneration (Sorensen et al., 2004). MMP-9 regulates cell differentiation during osteogenesis and angiogenesis, in part by the release of cytokines and growth factors from the extracellular matrix. In this study, MMP-9 was expressed in osteoclasts, and immunoblots were present in all the groups at all periods, similar to the untreated and TCP groups, thus indicating the level of enzyme activity of MMP-9 with respect to the similar, organic matrix-degradation process of bone remodeling. In the TCP + BMP-2 group, there was intense labeling of said enzyme in the 3 proposed periods, which may suggest that BMP-2 accelerates the remodeling process but without increasing the mean amount of new bone and/or mineralization.

Thus, it is conceivable that the supplementation of TCP with BMP-2 has a beneficial effect on bone healing. In this experimental model, bone repair was considered normal. The limitations of this study provide a basis for future studies, which will assess whether TCP provides satisfactory results without supplementation of BMP2 in various experimental models that might translate into a more reliable, clinical situation. In addition, data on the administration of BMP-2 with increased titration and observation periods (greater than 30 days postoperatively) may extend the current results.

\section{Conclusions}

The study shows that the formation of collagen matrices reached the maximum level when the defects in rat calvaria were filled with pure TCP, resulting in increased bone formation and mineralization. The use of TCP as a carrier for BMP-2 reduced formation of extracellular matrices and increased osteoclasis, denoted by intense MMP-9, which suggests a faster remodeling process. Further studies should be conducted with TCP to unveil its osteoconductive potential, and a new titration of BMP-2 and other carriers should be tested to consistently aggregate the osteoinductive capacity in the dynamics of bone healing.

\section{Conflict of interest}

The authors declare that they have no conflict of interest.

\section{References}

Bai, B., Yin, Z., Xu, Q., Lew, M., Chen, Y., Ye, J., Wu, J., Chen, D., Zeng, Y., 2009. Histological changes of an injectable rhBMP-2/calcium phosphate cement in vertebroplasty of rhesus monkey. Spine 34, 1887-1892.

Bostrom, M., Lane, J.M., Berberian, W.B., Missri, A.A., Tomin, E., Weiland, A., Doty, S.B., Glaser, D., Rosen, V.M., 1995. Immunolocalization and expression of bone morphogenetic proteins 2 and 4 in fracture healing. J. Orthop. Res. 13, 357-363.

Boyne, P.J., James, R.A., 1980. Grafting of the maxillary sinus with autogenous marrow and bone. J. Oral Surg. 38, 613-616.

Brånemark, P.I., Lindstrom, J., Hallen, O., 1975. Reconstruction of the defective mandible. Scand. J. Plast. Reconstr. Surg. 9, 116-128.
Carlson, T.L., Lee, K.W., Pierce, L.M., 2013. Effect of cross-linked and non-cross-linked acellular dermal matrices on the expression of mediators involved in wound healing and matrix remodeling. Plast. Reconstr. Surg. 131 (4), 697-705.

Dohzono, S., Imai, Y., Nakamura, H., Wakitani, S., Takaoka, K., 2009. Successful spinal fusion by E. coli derived BMP-2- adsorbed porous beta-TCP granules: a pilot study. Clin. Orthop. Relat. Res. 467, 3206-3212.

Esposito, M., Grusovin, M.G., Coulthard, P., Worthington, H.V., 2006. The efficacy of various bone augmentation procedures for dental implants: a Cochrane systematic review of randomized controlled clinical trials. J. Oral Maxillofac. Implants 21 (5), 696-710.

Johnson, E.E., Urist, M.R., Finerman, G.A., 1988. Bone morphogenetic protein augmentation grafting of resistant femoral nonunions: a preliminary report. Clin. Orthop. Relat. Res. 230, 257-265.

Luvizuto, E.R., Tangl, S., Zanoni, G., Okamoto, T., Sonoda, C.K., Gruber, R., Okamoto, R., 2011. The effect of BMP-2 on the osteoconductive properties of beta-tricalcium phosphate in rat calvaria defects. Biomaterials 32 (15), 3855-3861.

Luvizuto, E.R., Queiroz, T.P., Margonar, R., Panzarini, S.R., Hochulli-Vieira, E., Okamoto, T., Okamoto, R., 2012. Osteoconductive properties of beta-tricalcium phosphate matrix, polylactic and polyglycolic acid gel, and calcium phosphate cement in bone defects. J. Craniofac. Surg. 23 (5), e430-3.

Maus, U., Andereya, S., Gravius, S., Ohnsorge, J.Á., Siebert, C.H., Niedhart, C., 2008. BMP-2 incorporated in a tricalcium phosphate bone substitute enhances bone remodeling in sheep. J. Biomater. Appl. 22 (6), 559-576.

Misch, C.E., 1987. Maxillary sinus augmentation for endosteal impants: organized alternative treatment plans. Int. J. Oral Implantol. 4, 49-58.

Proussaefs, P., Lozada, J., 2005. The use of intraorally harvested autogenous block grafts for vertical alveolar ridge augmentation: a human study. J. Periodontics Restor. Dent. 25 (4), 351-363.

Reddi, A.H., 1998. Role of morphogenetic proteins in skeletal tissue engineering and regeneration. Nat. Biotechnol. 16 (3), 247-252.

Rentsch, C., 2014. Healing properties of surface-coated polycaprolactone colactide scaffolds: a pilot study in sheep. J. Biomater. Appl. 28 (5), 654-666

Schliephake, H., Weich, H.A., Dullin, C., Gruber, R., Frahse, S., 2008. Mandibular bone repair by implantation of rhBMP-2 in a slow release carrier of polylactic acid-an experimental study in rats. Biomaterials 29, 103-110.

Seeherman, H.J., Azari, K., Bidic, S., Rogers, L., Li, X.J., Hollinger, J.O., et al., 2006. rhBMP-2 delivered in a calcium phosphate cement accelerates bridging of critical-sized defects in rabbit radii. J. Bone Joint Surg. Am. 88, 1553-1565.

Sohier, J., Daculsi, G., Sourice, S., de Groot, K., Layrolle, P., 2010. Porous beta tricalcium phosphate scaffolds used as a BMP-2 delivery system for bone tissue engineering. J. Biomed. Mater. Res. A 92, 1105-1114.

Sorensen, R.G., Wikesjo, U.M., Kinoshita, A., Wozney, J.M., 2004. Periodontal repair in dogs: evaluation of a bioresorbable calcium phosphate cement (Ceredex) as a carrier for rhBMP-2. J. Clin. Periodontol. 31, 796-804

Tatum, H., 1986. Maxillary and sinus implant reconstructions. Dent. Clin. N. Am. 30, 207-229.

Tessier, P., Kawamoto, H., Matthews, D., Posnick, J., Raulo, Y., Tulasne, J.F., Wolfe, S.A., 2005. Autogenous bone grafts and bone substitutes-tools and techniques: I A 20,000 case experience in maxillofacial and craniofacial surgery. Plast. Reconstr. Surg. 116 (Suppl. 5), 6S-24S, discussion 92S-94S.

Toriumi, D.M., Robertson, K., 1993. Bone inductive biomaterials in facial plastic and reconstructive sugery. Facial Plast. Surg. 9, 29-36.

Van Den Bergh, J.P., Ten Bruggenkate, C.M., Groeneveld, H.H., Burger, E.H., Tuinzing, D.B., 2000. Recombinant human bone morphogenetic protein-7 in maxillary sinus floor elevation surgery in 3 patients compared to autogenous bone grafts. A clinical pilot study. J. Clin. Periodontol. 27, 627-636.

Wada, K., Niimi, A., Watanabe, K., Sawai, T., Ueda, M., 2001. Maxillary sinus floors augmentation in rabbits: a comparative histomorphometric study between rhBMP-2 and autogenous bone. J. Periodontics Restor. Dent. 21 (3), 252-263.

Wozney, J.M., Rosen, V., 1998. Bone morphogenetic protein and bone morphogenetic protein gene family in bone formation and repair. Clin. Orthop. Relat. Res. 346, 26-37.

Yao, J., Radin, S., Leboy, P.S., Ducheyne, P., 2005. The effect of bioactive glass content on synthesis and bioactivity of composite poly (lactic-co-glycolic acid)/bioactive glass substrate for tissue engineering. Biomaterials 26 , 1935-1943.

Yun, J.I., Wikesjo, U.M., Borke, J.L., Bisch, F.C., Lewis, J.E., Herold, R.W., Swiec, G.D., Wood, J.C., McPherson 3rd, J.C., 2010. Effect of systemic parathyroid hormone (1-34) and a beta-tricalcium phosphate biomaterial on local bone formation in a critical-size rat calvarial defect model. J. Clin. Periodontol. 37, 419-426.

Zhao, J., Hu, J., Wang, S., Sun, X., Xia, L., Zhang, X., Zhang, Z., Jiang, X., 2010. Combination of beta-TCP and BMP-2 gene-modified bMSCs to heal critical size mandibular defects in rats. Oral Dis. 16, 46-54. 